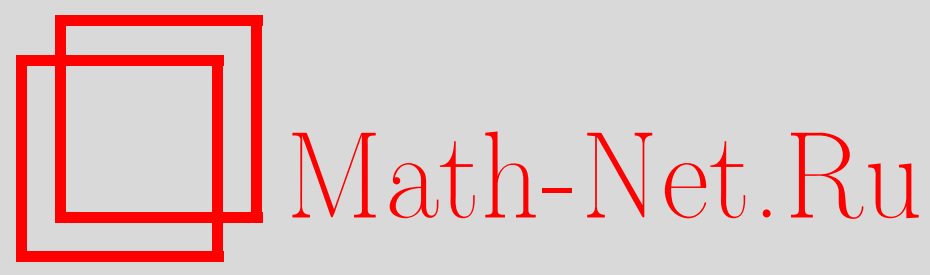

Б. И. Пелешенко, В. А. Катан, Об интегральных операторах свертки, Матем. заметки, 1999, том 66, выпуск 4, 551-555

DOI: https://doi.org/10.4213/mzm1198

Использование Общероссийского математического портала Math-Net.Ru подразумевает, что вы прочитали и согласны с пользовательским соглашением http://www. mathnet.ru/rus/agreement

Параметры загрузки:

IP: 54.174 .149 .18

26 апреля 2023 г., 14:40:47

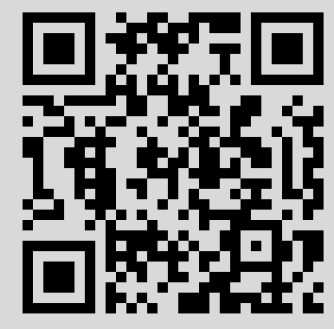


УДК 517.948.5

\section{ОБ ИНТЕГРАЛЬНЫХ ОПЕРАТОРАХ СВЕРТКИ}

\section{Б. И. Пелешенко, В. А. Катан}

В статье исследуются интегралы свертки, определяемые на функциях $n$ переменных из симметричных пространств. Установлены новые аддитивные оценки среднего значения невозрастающей перестановки модуля интеграла свертки $\left(K^{*} f\right)^{* *}(t)$ на отрезке $[0, t]$ для любого $t>0$. В качестве применения полученных оценок доказана ограниченность интегрального оператора свертки, действующего из пересечения симметричных пространств в пространство Марцинкевича.

Библиография: 4 названия.

В статье рассматриваются интегралы свертки

$$
\left(K^{*} f\right)(x)=\int_{\mathbb{R}^{n}} K(x-y) f(y) d y
$$

определяемые для измеримых на $n$-мерном евклидовом пространстве $\mathbb{R}^{n}$ функций $K(x)$ и $f(x)$.

Обозначим через $E\left(\mathbb{R}^{n}\right)$ симметричное пространство функций, заданньх на $n$-мерном евклидовом пространстве $\mathbb{R}^{n}$, с фундаментальной функцией $\varphi_{E}(t)$, и через $E^{\prime}\left(\mathbb{R}^{n}\right)$ пространство, ассоциированное с $E\left(\mathbb{R}^{n}\right)$.

Пусть $g^{*}(t)$ - невозрастающая перестановка модуля измеримой на $\mathbb{R}^{n}$ функции $g(x)$, $\chi_{[a, b]}(t)$ - характеристическая функция отрезка $[a, b]$,

$$
g^{* *}(z)=z^{-1} \int_{0}^{z} g^{*}(t) d t
$$

Множество непрерывных, положительных и неубьвающих на полуоси $(0, \infty)$ функций $\varphi(t)$, для которых $\varphi(0+)=0, \varphi(\infty)=\infty$, обозначается через $\Phi$. Для любой функции $\varphi(z) \in \Phi$ и $s>0$ определяется функция растяжения

$$
M_{\varphi}(s)=\sup _{z>0} \frac{\varphi(s z)}{\varphi(z)} .
$$


Теорема 1. Пусть невозрастающая перестановка $K^{*}(t)$ ядра оператора (1) интегрируема на отрезке $[0,1]$ u $K^{*}(t) \chi_{[1, \infty]}(t)$ принадлежит пространству $E(\mathbb{R})$. Тогда для любых функиий $f(x) \in E\left(\mathbb{R}^{n}\right), \delta(z) \in \Phi$ и любого $t>0$ имеет место неравенство

$$
\left(K^{*} f\right)^{* *}(t) \leqslant 2\left(\int_{0}^{\delta(t)} K^{*}(z) d z \cdot f^{* *}(t)+\left\|K^{*} \chi_{[\delta(t), \infty)}\right\|_{E^{\prime}(\mathbb{R})} \cdot\|f\|_{E\left(\mathbb{R}^{n}\right)}\right)
$$

ДокАЗАТЕЛЬСТво. Пусть функция $\delta(z) \in \Phi$ и $t>0$. Для произвольной функции $f(x) \in E\left(\mathbb{R}^{n}\right)$ и ядра интегрального оператора свертки, удовлетворяющего условиям теоремы, из неравенства О'Нейла [1] в случае $\delta(t)<t$ получаем

$$
\begin{aligned}
\left(K^{*} f\right)^{* *}(t) & \leqslant t K^{* *}(t) f^{* *}(t)+\int_{1}^{\infty} K^{*}(z) f^{*}(z) d z \\
& \leqslant \int_{0}^{\delta(t)} K^{*}(z) d z \cdot f^{* *}(t)+\int_{\delta(t)}^{t} K^{*}(z) d z \cdot f^{* *}(t)+\int_{t}^{\infty} K^{*}(z) f^{*}(z) d z
\end{aligned}
$$

Используя предположение теоремы о ядре $K(x)$ и неравенство типа Гёльдера для симметричных пространств [2], имеем

$$
\begin{gathered}
\int_{\delta(t)}^{t} K^{*}(z) d z \cdot f^{* *}(t) \leqslant\left\|K^{*} \chi_{[\delta(t), \infty)}\right\|_{E^{\prime}(\mathbb{R})} \cdot \varphi_{E}(t) \varphi_{E^{\prime}}(t) \cdot \frac{1}{t}\left\|f^{*} \chi_{[0, t]}\right\|_{E(\mathbb{R})}, \\
\int_{t}^{\infty} K^{*}(z) f^{*}(z) d z \leqslant\left\|K^{*} \chi_{[t, \infty)}\right\|_{E^{\prime}(\mathbb{R})} \cdot\left\|f^{*} \chi_{[t, \infty)}\right\|_{E(\mathbb{R})} \cdot
\end{gathered}
$$

Подставляя эти неравенства в $(3)$ и учитьвая, что $\varphi_{E}(t) \varphi_{E^{\prime}}(t) \leqslant t$, получим

$$
\left(K^{*} f\right)^{* *}(t) \leqslant \int_{0}^{\delta(t)} K^{*}(z) d z \cdot f^{* *}(t)+2\left\|K^{*} \chi_{[\delta(t), \infty)}\right\|_{E^{\prime}(\mathbb{R})} \cdot\|f\|_{E\left(\mathbb{R}^{n}\right)} .
$$

Для $\delta(t) \geqslant t$ из неравенства О'Нейла следует

$$
\begin{aligned}
\left(K^{*} f\right)^{* *}(t) & \leqslant t K^{* *}(t) f^{* *}(t)+\int_{t}^{\delta(t)} K^{*}(z) f^{*}(z) d z+\int_{\delta(t)}^{\infty} K^{*}(z) f^{*}(z) d z \\
& \leqslant 2 \int_{0}^{\delta(t)} K^{*}(z) d z \cdot f^{* *}(t)+\left\|K^{*} \chi_{[\delta(t), \infty)}\right\|_{E^{\prime}(\mathbb{R})} \cdot\|f\|_{E\left(\mathbb{R}^{n}\right)} .
\end{aligned}
$$

Теорема доказана. 
ТЕоРема 2. Пусть вогнутые функиии $\varphi(z), \psi(z)$ из множества $\Phi$ таковы, ито

$$
\int_{0}^{1} \frac{d z}{\varphi(z)}+\int_{1}^{\infty} \frac{d \psi(z)}{\varphi(z)}<\infty
$$

u $E\left(\mathbb{R}^{n}\right)$ - симметричное пространство с фундаментальной функиией $\varphi_{E}(t)=$ $t / \psi(t)$. Если невозрастающая перестановка ядра $K(x)$ оператора (1) удовлетворяет условию

$$
\sup _{t>0} K^{*}(t) \varphi(t)<\infty
$$

то для любых функиий $f(x) \in E\left(\mathbb{R}^{n}\right), \delta(z) \in \Phi$ и произвольного $t>0$ имеет место неравенство

$$
\left(K^{*} f\right)^{* *}(t) \leqslant C\left(\frac{\psi(t)}{t} \int_{0}^{\delta(t)} \frac{d z}{\varphi(z)}+\int_{\delta(t)}^{\infty} \frac{d \psi(z)}{\varphi(z)}\right) \cdot\|f\|_{E\left(\mathbb{R}^{n}\right)} .
$$

Здесь постоянная $C$ не зависит от $f(x), \delta(t), t$.

ДоКАЗАТЕЛЬСтво. Из условий теоремы 2 вытекает выполнение условий теоремы 1. Применяя неравенство (4), из теоремы 1 получим

$$
\left(K^{*} f\right)^{* *}(t) \leqslant C\left(\frac{1}{t} \int_{0}^{\delta(t)} \frac{d z}{\varphi(z)} \int_{0}^{t} f^{*}(z) d z+\left\|\frac{\chi_{[\delta(t), \infty)}(\cdot)}{\varphi(\cdot)}\right\|_{E^{\prime}(\mathbb{R})} \cdot\|f\|_{E\left(\mathbb{R}^{n}\right)}\right) .
$$

Далее оцениваем интеграл с помошью неравенства типа Гёльдера для симметричных пространств:

$$
\int_{0}^{t} f^{*}(z) d z \leqslant \varphi_{E^{\prime}}(t)\|f\|_{E\left(\mathbb{R}^{n}\right)}=\psi(t)\|f\|_{E\left(\mathbb{R}^{n}\right)} .
$$

Из вложения пространства Лоренща в симметричное пространство с той же фундаментальной функцией [2] получаем

$$
\left\|\frac{\chi_{[\delta(t), \infty)}(\cdot)}{\varphi(\cdot)}\right\|_{E^{\prime}(\mathbb{R})} \leqslant \int_{\delta(t)}^{\infty} \frac{d \psi(z)}{\varphi(z)} .
$$

Подставляя эти неравенства в неравенство (5), завершаем доказательство теоремы 2.

ТЕОРема 3. Пусть вогнутая функиия $\varphi(z)$ из множества $\Phi$ такова, что

$$
\int_{0}^{1} M_{\varphi}\left(\frac{1}{z}\right) d z+\int_{1}^{\infty} M_{\varphi}\left(\frac{1}{z}\right) \frac{d z}{z}<\infty,
$$

и для вогнутой фундаментальной функиии симметричного пространства $E\left(\mathbb{R}^{n}\right)$ выполняется неравенство

$$
\int_{1}^{\infty} M_{\varphi}\left(\frac{1}{z}\right) d\left(z M_{\varphi_{E}}\left(\frac{1}{z}\right)\right)<\infty .
$$

Если невозрастающая перестановка ядра $K(x)$ удовлетворяет условию (4), то для любых функиий $f(x) \in E\left(\mathbb{R}^{n}\right), \delta(z) \in \Phi$ и любого $t>0$ справедливо неравенство

$$
\left(K^{*} f\right)^{* *}(t) \leqslant C\left(\frac{\delta(t)}{\varphi(\delta(t))} f^{* *}(t)+\frac{\delta(t)}{\varphi(\delta(t)) \varphi_{E}(\delta(t))}\|f\|_{E\left(\mathbb{R}^{n}\right)}\right) .
$$

Здесь постоянная $C$ не зависит от $f(x), \delta(t), t$. 
ДокАЗАТЕЛьСтво. Сначала докажем, что интеграл

$$
\int_{0}^{\delta(t)} K^{*}(z) d z
$$

существует, и оценим его. Используя условие конечности интеграла

$$
\int_{0}^{1} M_{\varphi}\left(\frac{1}{z}\right) d z
$$

имеем

$$
\begin{aligned}
\int_{0}^{\delta(t)} K^{*}(z) d z & \leqslant C \frac{1}{\varphi(\delta(t))} \int_{0}^{\delta(t)} \frac{\varphi(\delta(z))}{\varphi(z)} d z=C \frac{\delta(t)}{\varphi(\delta(t))} \int_{0}^{1} \frac{\varphi(\delta(z))}{\varphi(s \delta(z))} d s \\
& \leqslant C \frac{\delta(t)}{\varphi(\delta(t))} \int_{0}^{1} M_{\varphi}\left(\frac{1}{s}\right) d s \leqslant C \frac{\delta(t)}{\varphi(\delta(t))} .
\end{aligned}
$$

Из вложения пространства Лоренца в симметричное пространство с той же (или эквивалентной) фундаментальной функцией [2] и условия (4) получаем

$$
\begin{aligned}
\left\|K^{*} \chi_{[\delta(t), \infty)}\right\|_{E^{\prime}(\mathbb{R})} & \leqslant \int_{\delta(t)}^{\infty} K^{*}(z) d \varphi_{E^{\prime}}(z) \leqslant C \frac{\varphi_{E^{\prime}}(\delta(t))}{\varphi(\delta(t))} \int_{\delta(t)}^{\infty} \frac{\varphi(\delta(z))}{\varphi(z)} d\left(\frac{\varphi_{E^{\prime}}(z)}{\varphi_{E^{\prime}}(\delta(z))}\right) \\
& \leqslant C \frac{\varphi_{E^{\prime}}(\delta(t))}{\varphi(\delta(t))} \int_{1}^{\infty} \frac{\varphi(\delta(z))}{\varphi(s \delta(z))} d\left(s \frac{\varphi_{E^{\prime}}(z)}{\varphi_{E^{\prime}}(s \delta(z))}\right) \\
& \leqslant C \frac{\delta(t)}{\varphi(\delta(t)) \varphi_{E^{\prime}}(\delta(t))} \int_{1}^{\infty} M_{\varphi}\left(\frac{1}{s}\right) d\left(s M_{\varphi_{E}}\left(\frac{1}{s}\right)\right)
\end{aligned}
$$

Так как по условию интеграл

$$
\int_{1}^{\infty} M_{\varphi}\left(\frac{1}{s}\right) d\left(s M_{\varphi_{E}}\left(\frac{1}{s}\right)\right)
$$

существует, из (6) следует, что

$$
\left\|K^{*} \chi_{[\delta(t), \infty)}\right\|_{E^{\prime}(\mathbb{R})} \leqslant C \frac{\delta(t)}{\varphi(\delta(t)) \varphi_{E^{\prime}}(\delta(t))} .
$$

Для завершения доказательства теоремы 3 подставим полученные оценки в неравенство $(2)$.

Пусть $\mathcal{M}_{\varphi}\left(\mathbb{R}^{n}\right)$ - пространство Марцинкевича функций $n$ переменных.

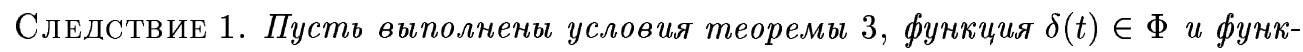
u,uu

$$
\psi_{1}(t)=\frac{\delta(t)}{\varphi(\delta(t)) \varphi_{E}(\delta(t))}, \quad \varphi_{1}(t)=\varphi(\delta(t))
$$

являются вогнутыми. Тогда для любой функиии $f \in \mathcal{M}_{\varphi_{1}}\left(\mathbb{R}^{n}\right) \cap E\left(\mathbb{R}^{n}\right)$ выполняется неравенство

$$
\left\|K^{*} f\right\|_{\mathcal{M}_{\psi_{1}}\left(\mathbb{R}^{n}\right)} \leqslant C\|f\|_{\mathcal{M}_{\varphi_{1}}\left(\mathbb{R}^{n}\right) \cap E\left(\mathbb{R}^{n}\right)} .
$$

Здесь постоянная $C$ не зависит от $f(x), \delta(t)$. 
СлЕдСТВИЕ 2. При выполнении условий теоремы 3 существует постоянная $C>0$ такая, что для любой функиии $f(x) \in E\left(\mathbb{R}^{n}\right)$ и произвольных $\alpha>0$ и $t>0$ выполняется неравенство

$$
\left(K^{*} f\right)^{* *}(t) \leqslant C\left(\frac{\alpha}{\varphi(\alpha)} f^{* *}(t)+\frac{\alpha}{\varphi(\alpha) \varphi_{E}(\alpha)}\|f\|_{E\left(\mathbb{R}^{n}\right)}\right) .
$$

Доказательство получаем из теоремы 3 ,выбрав для каждого $t>0$ функцию $\delta(z) \in \Phi$, значение которой в $t$ равно $\alpha$.

Установленные в статье оценки дополняют соответствуюшие результаты О'Нейла [1], Крейна, Петунина, Семёнова [2], Павлова [3] и др. Утверждение следствия 2 доказано Стефано [4] для операторов дробного интегрирования $K(x)=|x|^{\alpha-n}, 0<\alpha<n$, в пространствах $L_{p}\left(\mathbb{R}^{n}\right), 1<p<\infty$.

\section{СПИСОК ЦИТИРОВАННОЙ ЛИТЕРАТУРЫ}

[1] O'Neil R. Convolution operators and $L(p, q)$ spaces // Duke Math. J. 1963. V. 30. P. 129-142.

[2] Крейн С. Г., Петунин Ю. И., Семёнов Е. М. Интерполяция линейных операторов. М.: Наука, 1978.

[3] Павлов Е. А. Об интеграљьных операторах свертки // Матем. заметки. 1985. Т. 38. №1. C. $74-79$.

[4] Stefano M. A note on fractional integratio // Rend. Sem. Mat. Univ. Padova. 1989. P. 31-35. 